\title{
Improved Image Analysis Technique for Embedded System Design
}

\author{
Tamanna Afroze \\ Graduate Student, Computer Science Department \\ University of Texas at San Antonio \\ Texas, 78249-0667, USA
}

\begin{abstract}
Security is the primitive issue for humanity. From Paleolithic age, people are trying to secure themselves from natural calamity, furious animals and other adversities. With the advancement of technology, we get Machine gun, Nuclear Bomb, and many such powerful types of equipment for security. All these powerful equipments are used for protecting the border of a country, or in war against the enemy nation. General people cannot afford those powerful equipments. They need some simple but useful equipment necessary for protection against their enemies, which are wild enemies, cockroach, ants, and spiders. While working in a project of designing a device which will take care of the security issue for the general people, I need to design the image matching algorithm for the device named as Environment Aware Protection Device, in which whole detection mechanism of the insects is embedded in a chip of the device. An improved image analysis technique is used in this paper for detecting the bugs. Positional existence of organs within the insects is considered for image analysis
\end{abstract}

\section{General Terms}

Pattern Recognition, Security, Algorithms.

\section{Keywords}

Embedded system, security, cluster, feature set

\section{INTRODUCTION}

Image analysis is an interesting research area in both graphics and in computer architecture. In this paper, I have done detailed analysis on image for designing an efficient way of storing and comparing images within a device. Especially, when the device is an embedded system it needs much care in processing information stored in that device. The important factors that must be considered for any given application include performance, reliability, and cost. Any computer that handles large amount of graphical information, involving pictorial images and animation, must have good real time performance. In the case of personal computers and workstations, it is important to achieve the best performance for a cost that meets the demands of the marketplace. There are some applications, which do not need high performance processors or computer. Microprocessor controlled computational device are widely used for building many devices. Low cost and high reliability are essential factors for those devices than high performance. As cost issues are considered mainly for the microprocessor-controlled device, storing images; efficiently but having good performance is a challenging issue. Rest of the paper discusses the design issues for embedded system design, proposed design and performance of the proposed design.

\section{DESIGN ISSUE}

The designer of an embedded system has to make many important decisions. The nature of the application or the product that has to be designed presents certain requirements and constraints [1]. In this section, some important issues that designers face are going to be discussed briefly.

\subsection{Cost}

The cost of electronics in many embedded applications has to be low. The cheapest solution is realized if a single microcontroller chip is used for the whole application.

\subsection{I/O Capability}

Microcontroller chip provides variety of I/O lines. The number of $\mathrm{I} / \mathrm{O}$ lines available for use is an important matter for the design. These I/O lines vary from single to parallel I/O lines.

\subsection{Size}

Microcontroller chips come in variety of sizes. If an application can be handled with an 8-bit microcontroller, than it is useless to use a 16-bit microcontroller which ultimately consume more power, will be larger in size and will be expensive. After reading [2] and [3], I am using PIC microcontroller for my design.

\subsection{Power Consumption}

Power consumption is an important issue in designing device. In many embedded system, consumed power is low enough. Most of them may be battery-powered device. Microcontroller, which uses CMOS technology, consumes low power. Therefore, in the design of an embedded system it is necessary to choose a microcontroller, which consumes low power but maintains the performance level high.

\subsection{On-Chip Memory}

Microcontrollers often come with on-chip memory. For high volume data, handling device microcontroller with on-chip memory is used. For greatest flexibility, EEPROM and flash memories are used which can be programmed multiple times. In this design of my device, both external and on-chip memory is used. External EPROM is used to store captured images.

\subsection{Performance}

Performance is not a big issue when microcontroller is used for household devices. However, in applications which requires real-time applications performance is a great issue. So, depending on application microcontroller should be chosen smartly.

\subsection{Software}

There are many advantages to using high-level languages for computer application programs. Assembly language can also be used which produces 10 to 20 percent more compact code' (in terms of the storage 
needed).If an embedded application is based on a microcontroller that has on-chip memory, it is a major advantage if the necessary code can fit into the memory provided on the chip, avoiding the need for external memory.

\subsection{Instruction Set}

Another significant issue is the nature of the instruction set of the processor used. CISC-like instructions lead to more compact code than RISC-like instructions. Thus, a microcontroller should also be chosen carefully for meeting the desired need for the application which also meets the perfect instruction set.

\subsection{Development tools}

In many applications, many development tools are used. These computer aided design tools are used for designing the device. The range and availability of the design tools depends on embedded processor. These tools include operating system software, compiler, assembler, and simulator for the processor. In my design, I used MPLAB's IDE for simulating my device's performance [4].

\subsection{Testability and Reliability}

Printed circuit boards are often difficult to test, particularly if they are densely populated with chips. The testing process is greatly simplified if the entire system is designed to be easily testable.

\section{IMAGE ANALYSIS}

\subsection{Proposed Design}

Embedded system design is a challenge especially when devices have limited memory. For these systems, comparing two images is computationally expensive. Storing the whole image's information and then compare these two images is a big deal. Although logarithmic algorithm gives us a better and computationally inexpensive technique for matching a small picture in a large scene, we need to match the whole image and for embedded system, which uses microcontroller for all computational works the total picture comparison is almost impossible. Because microcontroller has limited number of registers and its size of EEPROM and programmable memory is very small. My proposal is to match and differentiate two images based on some specific features in a specific position of the object. Clustering algorithm [6] clusters particles, objects in a specific group. To differentiate two objects of a specific group we again need to match two objects wholly. Without matching two objects wholly, we can find out some unique feature in the object and by comparing their positional existence, we can distinguish two objects and simultaneously reduce the computational complexity. According to [5], presence or absence of any feature can be used to identify an animal's genus group. For example, presence or absence of lungs can be used to identify the category of the mammal, whether the mammal lives in sea or in land. Now, if we can find out a specific feature for all mammal lives in the sea we can use that feature's positional existence or size to distinguish two mammals. For my test we use two insects from arthropod, one is cockroach and another is spider. Both the cockroach and spider contain a sensory element in front of their head. For cockroach, its length is almost 2 inches, but for spider, it is almost half inches. It is a unique feature to distinguish these two objects for matching. I did not do any edit distance [7] or difference calculation for matching of two pictures. Matching is simply based on the color components of the pixel in an image. In brief, my proposal is to identify specific feature set, $\mathrm{F}=\{\mathrm{A}$, B \} for a particular group of insects where A, B are features of the original image and depending on the feature, images are compared. These feature set may vary in number of elements depending on the group of object. For example, in case of arthropod I used the sensory element and feature set, $\mathrm{F}_{\text {arthropod }}=\{$ sensory organ's positional existence $\}$. In order to differentiate object, at first, I need to group those objects depending on the biological feature, such as, arthropod, mollusk, mammal etc. Then I find out some specific feature set for each group. Depending on the specific feature, objects will be recognized. There is no preprocessing or post processing of feature sets. Unique features are selected without any modification. As the design is only developed based on feature selection without any modification, the model complexity is very low in this case. By model complexity, it is meant that the comparison of images and matching are not as much critical and complex for designing a low cost embedded system which has EEPROM's size limitation.

\subsection{Performance Analysis}

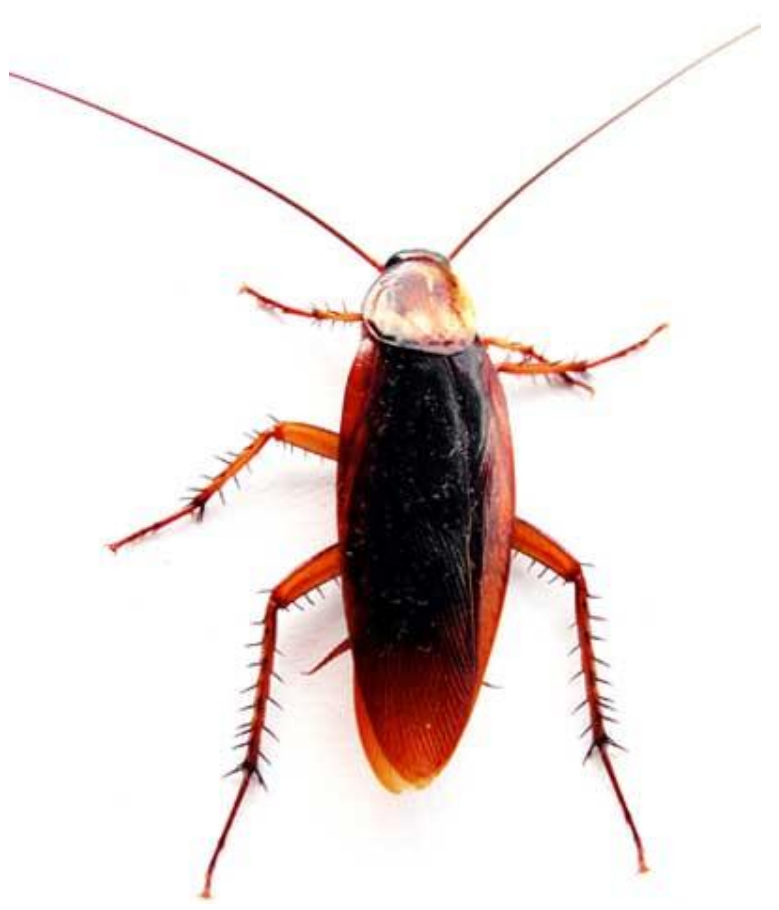

Figure 1: original.jpg

Matching algorithm is tested in MATLAB for correct matching. Figure 3 is the matching algorithm in MATLAB. Figure 1 is the original image for the arthropod group, which is cockroach, and the specific feature for this group is the presence of the sensory element, which is shown in Figure 2, named as part image. The output of the matching algorithm is shown in the Figure 4, which is the result of imshow(y). In fact, the algorithm is very simple. It reads the whole image and part of the image which is the feature set using imread () function. Then checks the existence of the feature set in the original image. If matching is found, then the value is stored 
in the $\mathrm{y}$ variable. The variable $\mathrm{y}$ is important for further computation. Because, the values of $y$ will go to the EEPROM of the microcontroller. The next simulation is tested in the MPLAB's simulator for testing using Programmable Interface Controller (PIC) microcontroller. The objective behind the device is the security of human being. Most of us hate insects like cockroach, spider. The protection device, namely, "Environment Aware protection Device" is targeted to sense the environment by capturing the image of the insects, detecting the hazardous insects using this matching algorithm to aware about the presence of an insect.

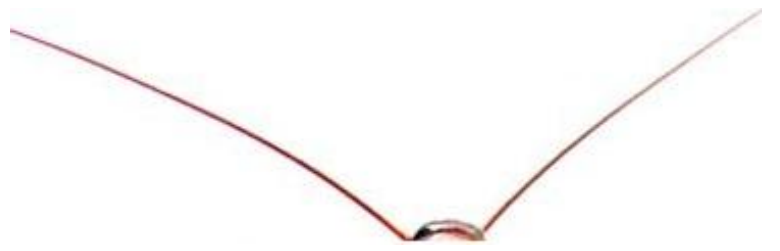

Figure 2: part.jpg

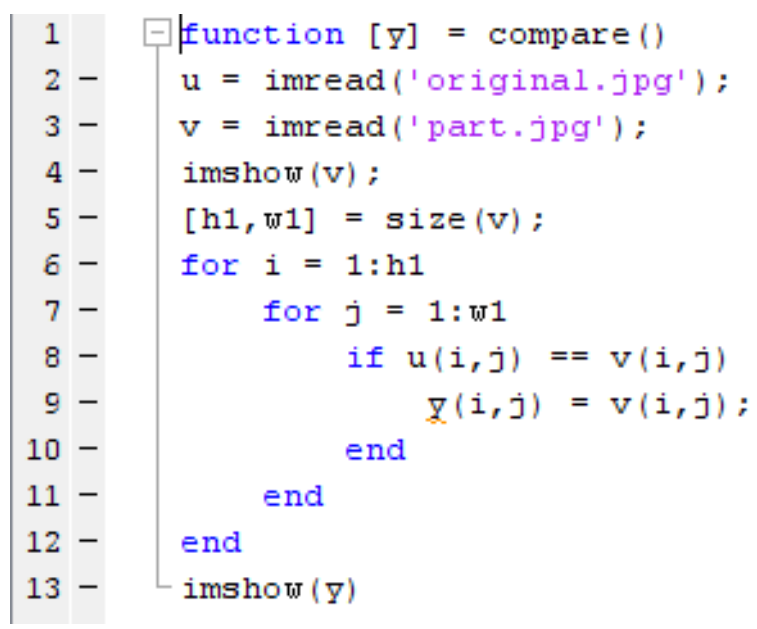

Figure 3: matching algorithm in MATLAB

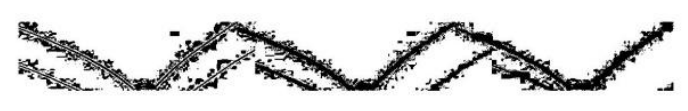

Figure 4: output generated by MATLAB after completion of matching

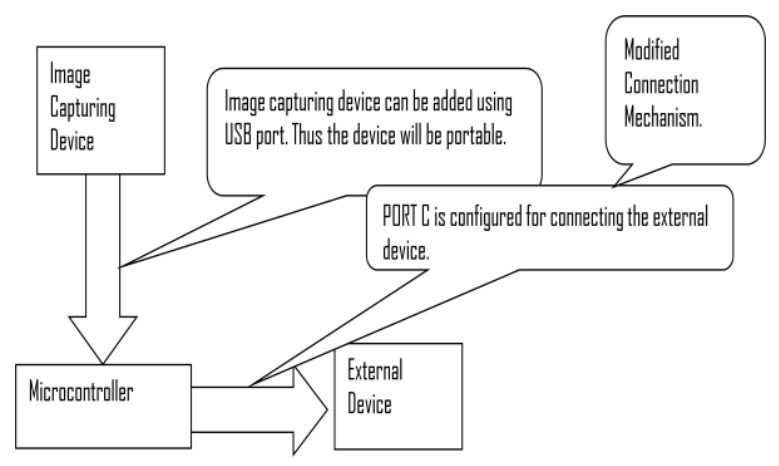

Figure 4: communication flow graph in the PIC Microcontroller
PIC18F8722 8-bit Microcontroller is used for implementing this device.The device is configured in MPASM environment with interrupt subroutines.Templates which are the feature sets, are stored in the device's EEPROM and captured image is also stored in the device EEPROM.After an image is captured interrupt subroutine is called and within the subroutine captured image is matched with all stored images. If matching is found necessary action is taken. The control flow graph of the whole mechanism is shown in the Figure 4 and Figure 5.Figure 5 is the detailed circuit diagram for the design, and Figure 4 is the main circuitry for the image processing.

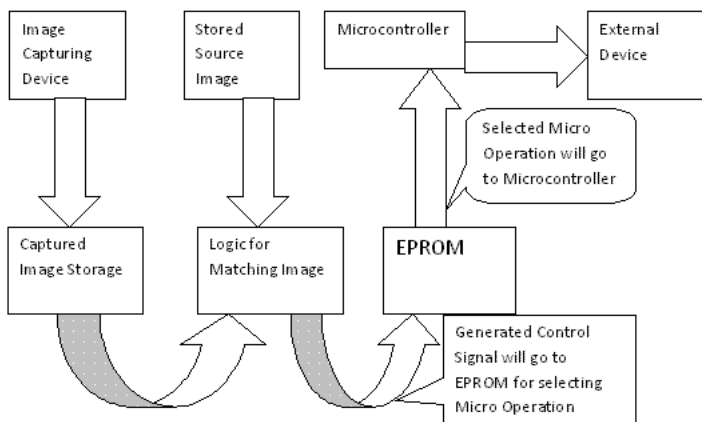

Figure 5: total circuit configuration flow graph for the PIC microcontroller.

Figure 6 shows the implementation in the MPLAB simulator. The EEPROM is shown where hexadecimal values of the partial image will be stored. In addition, that will be compared next for further matching. When matching is found interrupt subroutine will be called for and in interrupt subroutine initiates protection mechanism to start up. That will be hardware mechanism by which the device will protect human from hazard.

Running time of the algorithm in MATLAB is $O\left(n^{2}\right)$. Therefore, it may be think that this proposition is not an improved idea for analysis of image as we have logarithmic algorithm for image matching. In case of logarithmic algorithm, we have to compare the whole image, but in this algorithm, we do not compare the whole image, we only compare the partial image's existence in the whole image. Therefore, it is an improved mechanism of image analysis. In case of every image, we find out here a partial image, which is of very small size of the whole image. 


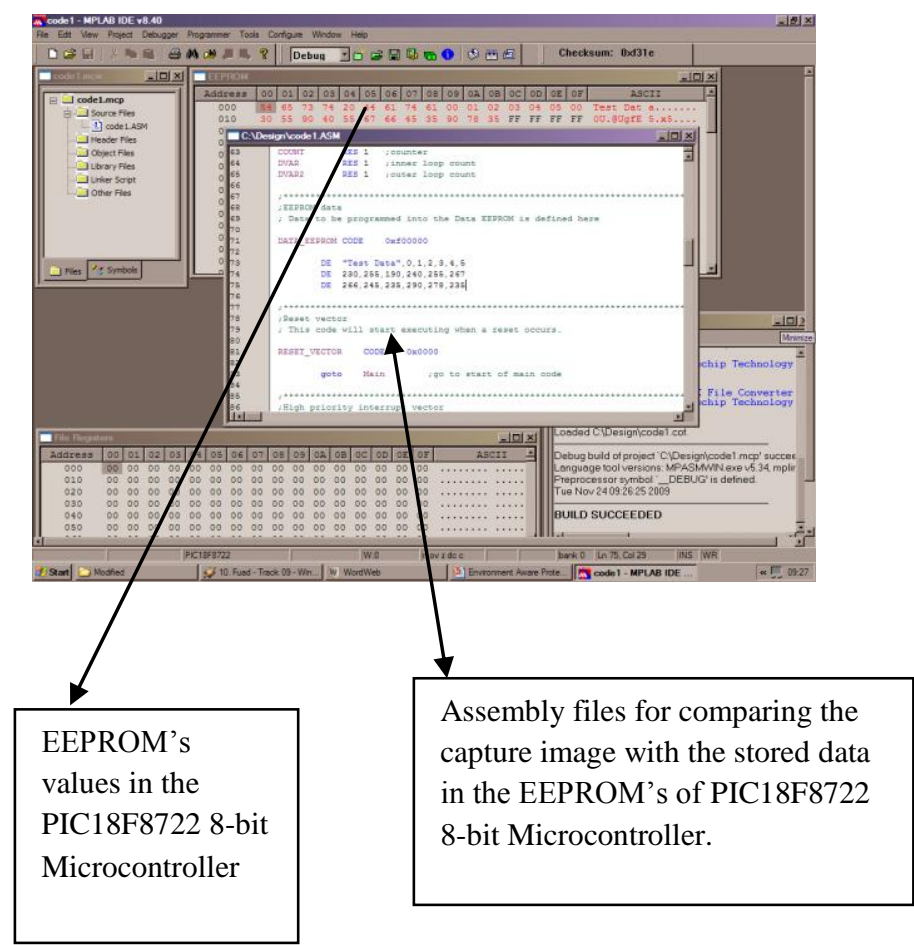

Figure 6: implementation of the proposed design in MPLAB simulator.

\section{CONCLUSION}

In this paper, proposition is made for image analysis in a different way for embedded system design. Images are considered in a new way, that is, images are grouped at first depending on some unique feature. The original picture is then compared to the unique feature for matching, and the result is stored in the EEPROM of the microcontroller. In the performance analysis section, it is mentioned that the original picture captured by the camera will be store in the EEPROM of the microcontroller. The microcontroller's EEPROM size is very small, and images will be store in hexadecimal format. In case of original implementation it will be tested whether image captured by the camera can be stored in camera's memory and then simulator will just compare the original image stored in the camera with the partial image stored in the EEPROM of the microcontroller.
Whole proposition is just based on simulation, two types of simulation is used here in this proposition. At first, MATLAB simulation is used to compare the original image with the partial image. If matching is found, then the partial image will be stored EEPROM of the simulator's microcontroller.

In this proposition, my intension is to develop a device for human being, which will protect them from hazardous insects. The device is named as Environment Aware Protection device, where there will be a camera embedded in the device with a microcontroller. Microcontroller has an EEPROM where partial image will be stored, and original image may or may not be stored in the original design.

In EEPROM, the image's hexadecimal values will be stored. That is shown in the figure in performance analysis section.

\section{ACKNOWLEDGEMENT}

My thanks to disguished reviewers for their many valuable comments. Also, my thanks goes to Louisiana State Universitie's courses during the session Fall 2009, and University of Texas at San Antonio's courses during Spring, 2013 session.

\section{REFERENCES}

[1] Hamacher Carl, Vranesic Zvonko, Zaky Safwat "Computer Organization." Mc Graw Hill, fifth edition, pp 516-549, 2002.

[2] Skroder C. John in "Using the M68HC11 Microcontroller - A Guide to Interfacing and Programming the M68HC11 Microcontroller”.Willey Publication

[3] Peatman B. John. In "Design With PIC Microcontroller", Second Edition.

[4] Mannual on PIC Microcontroller, Retrieved From www.support.microchip.com.

[5] Hubalek Z. "Coefficients of association and similarity based on binary (presence-absence) data - an evaluation," Biological Review, Vol. 57, pp.669-689, 1982

[6] Jain A.K., Dubes R.C. Algorithms for Clustering Data, Prentice Hall, 1988.

[7] Theodoridis S., Koutroumbas K. Pattern Recognition, Academic Press, 2003. 